\title{
Les réacteurs nucléaires de demain et d'après-demain
}

\section{Présentation}

par Bertrand BARRÉ, CEA

Les grandes célébrations du centenaire de la découverte de la radioactivité ont peut-être fait oublier à quel point l'utilisation pratique de l'énergie nucléaire est récente. Que ce fût à Obninsk ou Calder Hall - je me garderai de trancher - le premier réacteur "commercial" n'a divergé qu'il y a 45 ans environ, et le "programme Messmer" n'a que 25 ans. Se souvient-on encore qu'en 1974 la France n'avait que 8 \% d'électricité nucléaire?

En moins de 50 ans, l'énergie nucléaire est passée de 0 à $17 \%$ de l'électricité mondiale, et ces $17 \%-2400$ TWh - excèdent la production d'électricité de 1960.

Alors que les premières années d'après-guerre avaient connu un foisonnement incroyable de réacteurs différents, couvrant presque toutes les combinaisons possibles de matières fissiles, fertiles, de modérateurs et de fluides caloporteurs, les quelques décennies qui ont suivi ont vu à l'œuvre un processus féroce de sélection "darwinienne", avec très peu de familles survivantes : $85 \%$ des réacteurs aujourd'hui en opération sont des réacteurs à eau ordinaire (REP, REB et WER), deux autres familles n'ont pas de descendance (Magnox/AGR et RBMK), et seuls les réacteurs à eau lourde CANDU défendent encore une niche marginale, fondée sur I'utilisation d'uranium naturel, et la taille moyenne des unités, qui les rendent - disent leurs promoteurs - plus accessibles aux nouveaux entrants dans le monde nucléaire.

Les réacteurs à eau ordinaire sont robustes, fiables et beaucoup plus souples que ce qu'en attendaient leurs concepteurs. Dans des circonstances favorables, telles que celles du parc standardisé français, ces réacteurs restent compétitifs, en base, même en période de très bas prix des combustibles fossiles, mais seulement pour de très grandes tailles unitaires (1300 MWe et plus).

Pour atteindre le très haut niveau de sûreté exigé dans les pays industrialisés, les réacteurs à eau ordinaire doivent être soumis à une maintenance sans faille, et faire appel à des systèmes complexes de sauvegarde : leur maîtrise demande des équipes d'opérateurs de grande technicité et régulièrement entraînés.

Spécifiés dans les années 60 et 70 , les réacteurs à eau ordinaire (REO) sont toujours aujourd'hui ce qui se fait de mieux, résultat vraiment remarquable !

Mais, nous l'avons dit, l'énergie nucléaire est jeune, les techniques progressent, les besoins sociaux évoluent, les contextes changent.

- L'exigence, née de Tchernobyl, de ne jamais occasionner de relâchement massif de radioactivité dans l'environnement, même en cas de fusion complète du cœur, fait déjà d'EPR une machine assez différente des N4 et Convoy qui l'ont précédée.

- L'escalade de tailles unitaires, qui a permis au réacteur à eau ordinaire de garder sa compétitivité dans les pays les plus sophistiqués (France, Japon, Allemagne, etc.) en a, petit à petit, fait des machines peu accessibles aux débutants. Casser cet enchaînement nécessitera peut-être de changer radicalement de technologie. Changer de technologie contraindrait à reprendre "sur une base zéro" l'analyse de la sûreté des réacteurs, qui ne pourraient plus se prévaloir de l'énorme retour d'expérience des REP, mais repartir de la page blanche permettrait peut-être de tirer réellement parti de progrès inhérents dans la sûreté, en termes de simplification, et donc de coûts. C'est ce double espoir qui motive Framatome à rouvrir le dossier des réacteurs à haute température, les HTR, aux caractéristiques attrayantes, mais qui ont eu la malchance de tenter leur percée commerciale aux EtatsUnis en 1973 ; juste avant que ce pays n'annule toutes ses commandes de réacteurs.

- Les attentes du public - et de ses représentants - en matière de gestion des déchets radioactifs, et surtout des déchets de longue durée de vie, ont beaucoup augmenté depuis une quinzaine d'années. A l'heure qu'il est, aucun pays n'a encore autorisé l'exploitation, voire même la construction d'un site de stockage définitif des déchets de haute activité ou des déchets civils de longue durée de vie ${ }^{(1)}$. Beaucoup de pays étudient donc la faisabilité de transmuter tout ou partie des isotopes de longue durée de vie dans des réacteurs optimisés à cet effet. C'est dans ce cadre que se situent, notamment, les études (conceptuelles à ce stade) de réacteurs "hybrides", dans lesquels une source de spallation suralimente en neutrons un milieu multiplicateur sous-critique.

- Si la fission doit contribuer sur le long terme à l'approvisionnement énergétique de l'humanité - et on voit mal comment assurer un "développement durable" en son absence - il faudra un jour mettre en service des réacteurs plus sobres que les REO, qui ne tirent parti que de $1 \%$ environ du contenu énergétique de l'uranium. II faudra aussi assurer le vrai multirecyclage du plutonium (sans lequel, incidemment, la transmutation des actinides n'a guère de sens). Quelle sera la technologie de ces consommateurs d'Uranium 238 ? L'avenir nous le dira - mais ce seront des réacteurs à neutrons rapides.

Voici quelques-unes des libres réflexions que m'inspire le titre de ce numéro de la RGN "les réacteurs nucléaires de demain et d'après-demain", mais j'en reviens à mon propos initial : l'énergie de fission est jeune et, pour paraphraser un chroniqueur matutinal, son futur est plein d'avenir ! 